\title{
La educación inclusiva en docentes en formación: su evaluación a partir de la teoría de facetas
}

\author{
Inclusive education in teacher training: its evaluation \\ from the facet theory
}

Libia Vélez Latorre ${ }^{1}$

\author{
Resumen
}

El propósito del presente estudio fue identificar las actitudes que tienen los docentes en formación hacia la educación inclusiva. El diseño y validación del instrumento para evaluar las actitudes se hizo a partir del modelo de investigación de teoría de facetas y el análisis de la información se llevó a cabo por medio del análisis de espacio más pequeño (SSA) y de estadística descriptiva. Participaron de la investigación 91 estudiantes de los cuatro programas de Licenciatura de la Facultad de Educación de la Universidad Pedagógica Nacional. Los resultados muestran que las facetas propuestas en la frase mapa para evaluar las actitudes: medida de la actitud, agentes y escenarios educativos, y elementos didácticos, fueron confirmadas por el análisis SSA. En general, los docentes en formación muestran actitudes positivas hacia la educación inclusiva. Se identifica además que los participantes visualizan al docente como el agente educativo central de la educación inclusiva y consideran que para realizarla es indispensable contar con recursos o materiales especializados para el trabajo en el aula y un respaldo institucional para su consecución y la cualificación de los docentes. Finalmente se proponen tres ejes de formación necesarios para los futuros licenciados en aras de favorecer las actitudes hacia la educación inclusiva.

\section{Palabras claves:}

Actitudes, educación inclusiva, agentes y escenarios educativos, elementos didácticos, teoría de facetas.

\section{Abstract}

The purpose of this study was to identify the attitudes that prospective teachers have toward inclusive education. The design and validation of the instrument for assessing attitudes was approached from the facet theory model, and the analysis of the information was carried out by means of the smallest space analysis (SSA) and descriptive statistics. Ninety-one students from the four undergraduate programs of the Faculty of Education at the National Pedagogical University participated in the study. The results corroborate that the facets proposed in the mapping-sentence for evaluating attitudes: attitude components, agents and educational settings, and educational elements were validated by the SSA analysis. In general, teachers in training have positive attitudes toward inclusive education. The study also identifies that participants conceptualized the teacher as the central educational agent for inclusive education. They also believe that to achieve inclusive education it is necessary to have resources or specialized materials for work in the classroom and institutional support for its attainment and qualification of teachers. The article proposes three areas of training necessary for future graduates in order to promote attitudes towards inclusive education.

Keywords:

Attitudes, inclusive education, agents and educational settings, educational elements, facets theory.

Artículo recibido el 15 de diciembre de 2012 y aprobado el 8 de febrero de 2013

1 Universidad Pedagógica Nacional, Bogotá, Colombia. Correo electrónico: Ivelez@pedagogica.edu.co. 


\section{La educación inclusiva y la formación de los docentes}

La importancia de la educación inclusiva yace en que es un asunto de derechos humanos, de valores y creencias y una reivindicación del derecho de todos los estudiantes a recibir una educación con calidad. La educación inclusiva plantea la existencia de un solo sistema educativo, la institución debe transformarse para garantizar la atención educativa a todos, dando respuestas educativas y oportunidades de aprendizaje y participación a través de un currículo flexible y de los apoyos que aumentan la capacidad del entorno para responder a la diversidad (Vélez, 2011, p. 7).

La inclusión significa atender con calidad y equidad a las necesidades comunes y específicas que presentan los estudiantes (MEN, 2008, p. 10). Por su parte, la Unesco (2009) define la educación inclusiva como

un proceso que comporta la transformación de las escuelas y de otros centros de aprendizaje para atender a todos los niños - esto es, los niños y las niñas, los alumnos que pertenecen a grupos étnicos y lingüísticos minoritarios o a poblaciones rurales, aquellos afectados por el VIH y el sida o con discapacidad y dificultades de aprendizaje-, y para brindar también oportunidades de aprendizaje a todos los jóvenes y adultos. Tiene por objetivo acabar con la exclusión que es consecuencia de actitudes negativas y de una falta de atención a la diversidad en materia de raza, situación económica, clase social, origen étnico, idioma, religión, sexo, orientación sexual y aptitudes (p. 5).

Al respecto Vélez (2011) afirma:

La educación inclusiva se enmarca en un modelo social, a partir del cual las barreras para el aprendizaje y la participación están en el entorno, siendo este el responsable de minimizarlas y/o abolirlas. Las dificultades están fundamentalmente en el currículo, no en el estudiante. Las implicaciones educativas tienen que ver con la valoración de la diversidad como condición humana y con la diferencia como un aspecto inherente a todos los seres humanos, por ello, todos los sujetos se entienden como diversos, diferentes. La responsabilidad del proceso educativo es de toda la comunidad educativa. La educación inclusiva supone entonces un cambio social en la manera de concebir las prácticas, las culturas y las políticas educativas y escolares (p. 7).

El concepto de educación inclusiva pone en tela de juicio gran parte del modo de organizar y disponer la enseñanza de las escuelas tradicionales. Aunque las escuelas deben tener objetivos generales o comunes en cuanto a lo que es adecuado y deseable que los educandos logren en ellas, las demandas relacionadas con las distintas materias escolares se deben considerar en el contexto de las oportunidades y necesidades de cada educando (Unesco, 2009, p. 20).

Es importante recalcar que esta concepción de la educación para todos no está reservada solo para personas con necesidades educativas especiales asociadas o no a una discapacidad, sino para todos los niños y jóvenes; desde el principio de una educación con equidad, la educación inclusiva valoriza las capacidades de desarrollo, aprendizaje y participación de todos, es una herramienta para superar la pobreza, disminuir la exclusión escolar, reducir las desigualdades sociales, promover la integración cultural y social. Esta perspectiva también reconoce que los seres humanos están en permanente interacción con contextos que facilitan o ponen barreras actitudinales, culturales, políticas, físicas, técnicas, tecnológicas y de prácticas pedagógicas que promueven o impiden su desarrollo integral.

\section{$[\ldots]$}

Tradicionalmente los docentes han sido formados para la homogeneidad, desde representaciones de estudiantes que son uniformes en sus maneras de aprender y construir conocimiento. La formación entonces no ha estado basada en la noción de seres humanos heterogéneos, diversos, que tienen sus propios ritmos y estilos de aprendizaje y, distintos tiempos y modos en su desarrollo y que por ello precisan de diferentes formas de enseñanza, diferentes usos de estrategias didácticas, meto- 
dológicas. Es el caso de la Universidad Pedagógica Nacional y en general de las universidades y facultades de educación del país formadoras de docentes, la formación se ha orientado fundamentalmente al desarrollo de su labor desde un paradigma ideológico homogeneizador, que no reconoce la diversidad como condición y valor de los seres humanos, sino como una imposibilidad - unas veces- o una dificultad -en otras ocasiones- para ser, tener, hacer y estar. (Vélez, 2011, p. 7)

En consecuencia, los docentes en ejercicio han visto aún más insuficiente su propia formación para atender la diversidad de estudiantes en las instituciones educativas y lo que es más delicado y trascendental, gran parte de ellos continúan considerando que la atención a todos, que la atención a la diversidad no es un asunto que les compete porque no fueron formados para ello, porque fueron formados para una normalidad que no acoge la diversidad (Vélez, 2011, p. 8).

Los programas de formación de docentes, tanto iniciales como en el servicio, deberían reorientarse y estar armonizados con los planteamientos de la educación inclusiva para proporcionar a los docentes las competencias necesarias con miras a lograr que la diversidad sea beneficiosa en el aula y de conformidad con los programas de estudios reformados. (Unesco, 2009, p. 18).

La educación inclusiva es una tendencia educativa y social que hoy, más que nunca, ha hecho conciencia de las necesidades de transformación que requerirá avanzar en condiciones culturales, políticas y de prácticas favorables a procesos de inclusión. Las facultades de educación y en general las instituciones formadoras de docentes, no pueden ser ajenas a esta situación y deben tomar medidas en la formación de docentes, concebida como uno de los factores decisivos del sistema educativo y de la sociedad. Es necesario entonces que los docentes estén formados para la atención a diversidad y para la educación inclusiva (Vélez, 2011, p. 9).

\section{Importancia de la evaluación y formación de actitudes positivas hacia la inclusión}

Sola (1999, p. 389) señala algunos aspectos básicos que deben considerarse en la formación inicial de docentes con el propósito de lograr en esta actitudes positivas hacia la integración ${ }^{2}$. Entre los aspectos mencionados, el autor plantea la necesidad de implementar una formación coherente y bien planificada tanto inicial como permanente. Lo anterior supone que la falta de formación adecuada podría ocasionar desinterés y un abierto rechazo hacia la atención a la diversidad en la educación regular.

Por su parte Aguilar (2010) concluye en su estudio sobre la influencia de las actitudes de los docentes sobre el rendimiento académico de los estudiantes lo siguiente:

Los profesores en un principio general no siempre basan sus expectativas en evaluaciones objetivas y formales de cada alumno, más bien las construyen sobre impresiones formadas a partir de aspectos externos provenientes, en su mayoría, de la propia escuela o bien de la familia, su cultura, su sociedad. Cualquiera sea la procedencia, esta irá irremediablemente determinando el futuro comportamiento de cada alumno, por lo que se hace urgente, por parte de los profesionales de la educación, tomar conciencia sobre el poder transformador que las expectativas tienen sobre el proceso de enseñanza-aprendizaje y el rendimiento escolar. Será tarea de cada profesor reconocer las propias expectativas y transformar aquellas que sean dañinas para los alumnos (p. 6)

En consecuencia, las actitudes positivas hacia la diversidad y la inclusión se deben desarrollar desde la formación inicial de los docentes. Sales, Moliner y Sanchiz (2001), afirman:

Uno de los factores que influyen poderosamente en el desarrollo de modelos inclusivos de atención a la diversidad en las escuelas es el de las actitudes de la comunidad escolar: cómo se conceptualiza la diversidad, cómo se evalúa afectivamente, cómo se actúa ante ella (p. 2).

2 Aunque Sola hace referencia al proceso de integración, es posible realizar un símil en relación a las actitudes hacia la inclusión. 
Autores como Stainback y Stainback (1999), Alegre (2000), Arnáiz (2003) y Tilstone, Florian y Rose (2003), entre otros, coinciden en señalar que uno de los pilares para que la educación inclusiva sea efectiva es la formación de los docentes para atender las características diversas de los estudiantes, pues son ellos los que llevarán a cabo los cambios en las aulas y en las escuelas.

Se vuelca entonces la mirada hacia la formación de los futuros docentes. Lo que se requiere entonces, señalan Sales, Moliner y Sanchiz (2001),

es que el futuro docente desarrolle actitudes favorables hacia la diversidad del alumnado y se sienta capaz de buscar, reflexionar y consensuar junto con otros agentes educativos y sociales distintas alternativas, que pueden encontrarse en el contexto escolar, para actuar ante las necesidades educativas especiales ${ }^{3}$, considerando que el sistema educativo ha de proporcionar los servicios y recursos necesarios para que todos los alumnos puedan desarrollar al máximo sus capacidades en igualdad de oportunidades (p. 5).

En este sentido, para la educación inclusiva, educar para la diversidad, constituye uno de los aspectos clave de la formación inicial de los docentes, siendo el desarrollo de actitudes positivas hacia la diversidad del estudiantado una preocupación primordial en todos los programas de formación de docentes en el mundo.

La implementación de una estrategia educativa y de políticas orientadas en esta perspectiva requiere de una comunidad educativa preparada para aceptar la inclusión en todas las dimensiones del proceso educativo, lo cual ha dado lugar al diseño de políticas que han favorecido este proceso en otras partes del mundo. Sin embargo no se conocen estudios que evalúen y formen a los maestros en la inclusión en el caso colombiano, de ahí la importancia de conocer y evaluar las actitudes hacia la educación inclusiva en los docentes que actualmente se encuentran en

3 Pese a que el presente artículo no emplea el término NEE, puesto que en el contexto de la educación inclusiva se hace uso del término barreras para el aprendizaje y la participación, es importante citar el trabajo de este autor por su referencia a la formación adecuada de actitudes del profesorado ante la diversidad de estudiantes. las universidades e instituciones de educación superior del país y, particularmente, en la Universidad Pedagógica Nacional, institución responsable de la formación de la mayor parte de maestros del país.

De acuerdo con los anteriores planteamientos, para generar una propuesta de formación inicial de docentes hacia la Educación Inclusiva, es necesario partir del reconocimiento de las actitudes que ellos tienen actualmente. Para alcanzar este propósito, el presente estudio parte de la identificación del campo de las actitudes hacia la educación inclusiva desde la delimitación de un modelo de medición de actitudes que contiene los componentes de las actitudes, los agentes y escenarios educativos, y los elementos didácticos. Estos tres componentes surgen de la revisión de la literatura académica que ha logrado identificar que para el desarrollo exitoso de los procesos de inclusión, además de una disposición de los agentes educativos y la institución a aceptar a todos los integrantes del aula a partir de la diferencia y la diversidad humana; es necesario que los escenarios educativos cuenten con los elementos didácticos en términos de estrategias, recursos o materiales y ambientes de aprendizaje que garanticen las acciones de enseñanza, las herramientas o medios didácticos y, los escenarios y espacios curriculares que propicien y faciliten el aprendizaje y participación de todos los estudiantes.

Sobre la puesta a prueba de este modelo de evaluación de actitudes hacia la educación inclusiva se formulan recomendaciones para la formación de actitudes positivas en los estudiantes que se forman actualmente en las cuatro licenciaturas de la Facultad de Educación.

\section{Los componentes de las actitudes}

La mayoría de los investigadores consideran la actitud como una predisposición aprendida para actuar en forma favorable o desfavorable con respecto a la elección de un objeto a partir de tres elementos: cognoscitivo, afectivo e incitadores a la acción Fishbein y Ajzen (1975, p. 6). El elemento conductual (incitadores a la acción) identifica cómo actúa o actuaría el individuo con relación 
al entorno. El aspecto afectivo (emocional) es la posición afectiva hacia el entorno; sentimientos, preocupaciones, sensaciones, etc. El cognoscitivo se refiere a conocimientos y sistemas de creencias del individuo acerca de la situación real de su entorno. Independientemente de la conducta que presente el sujeto al momento de hacer su elección, le antecederá un conocimiento o creencia, una sensación o emoción, y un posible repertorio conductual. El evaluar estos tres componentes permite tener una visión amplia de las verdaderas disposiciones de los docentes en formación hacia la educación inclusiva. En consecuencia con esta información como punto de partida es posible plantear acciones que de forma articulada trabajen los tres componentes para lograr actitudes positivas hacia la educación inclusiva.

\section{Agentes y escenarios educativos}

Para el caso del modelo metodológico que se propone para evaluar las actitudes, se definen como agentes educativos el docente y los estudiantes, $y$ también los escenarios educativos que tienen que ver con el aula y la institución educativa; si bien el docente y los estudiantes son los agentes por excelencia en cualquier proceso educativo, el aula y la institución educativa se constituyen en el contexto que condiciona en gran medida los resultados de dicho proceso. Específicamente para este estudio, se establecieron los agentes y escenarios educativos indicados, los cuales fueron referidos como los más prioritarios por los docentes en formación que realizan sus prácticas en contextos inclusivos 4 .

\section{Elementos didácticos}

Se definen tres elementos didácticos: las estrategias, los recursos o materiales y los ambientes de aprendizaje los cuales se describen, brevemente, a continuación:

Estrategias: son las acciones de enseñanza realizadas por los docentes, para el caso de la educación inclusiva, soportadas en unos principios metodo-

4 Dichas prácticas se enmarcan en el desarrollo de la tesis doctoral "La educación inclusiva en los programas de formación inicial de docentes" (cf. Vélez, 2013). lógicos que propicien y faciliten el aprendizaje y la participación de todos los estudiantes del aula. En este sentido, las estrategias en un contexto de educación inclusiva, deben estar soportadas, desde lo pedagógico, en el conocimiento de cada uno de los estudiantes, en términos de una caracterización de los procesos de aprendizaje y participación, así como de las barreras y facilitadores para dichos procesos. Desde lo metodológico, las estrategias constituyen todas las oportunidades y formas usadas por el docente para disminuir, minimizar o abolir las barreras a las que se enfrentan los estudiantes para aprender y participar. Desde lo didáctico, las estrategias deben tener en cuenta las necesidades, intereses, expectativas y posibilidades de cada estudiante, y los diferentes procedimientos en cuanto a diseño, programación, realización y evaluación de los procesos de enseñanza y aprendizaje.

Recursos o materiales: son aquellas herramientas o medios didácticos que apoyan la realización de las actividades de aprendizaje planteadas por el docente, facilitando el acceso al conocimiento y el logro de los objetivos propuestos y, más específicamente, en el caso de la educación inclusiva, motivando el aprendizaje y participación de todos y cada uno de los estudiantes, teniendo en cuenta sus particularidades y capacidades.

Cebrián (citado por Cabero, 2001) define los recursos didácticos como

Todos los objetos, equipos y aparatos tecnológicos, espacios y lugares de interés cultural, programas o itinerarios medioambientales, materiales educativos que, en unos casos utilizan diferentes formas de representación simbólica, y en otros, son referentes directos de la realidad. Estando siempre sujetos al análisis de los contextos y principios didácticos o introducidos en un programa de enseñanza, favorecen la reconstrucción del conocimiento y de los significados culturales del currículum (p. 290).

Dentro de los principales recursos o materiales se encuentran los libros de textos, audiovisuales, juegos, software, equipos, sistemas de comunicación aumentativa y alternativa. 
Ambientes de aprendizaje: son los escenarios y espacios curriculares en los que el docente propicia y facilita el aprendizaje y la participación de todos los estudiantes. En un contexto de educación inclusiva, los ambientes de aprendizaje apuntan a las diferentes formas de organización del aula para disminuir, minimizar o abolir las barreras a las que se enfrentan los estudiantes en el contexto escolar. En este sentido deben incluir diferentes aspectos como: a) acceso al mobiliario: este debe tener condiciones físicas y ergonómicas necesarias para que los estudiantes puedan lograr adecuados niveles de concentración a lo largo de toda la jornada académica; b) acceso y movilidad a toda la planta física: todos los estudiantes deben poder acceder y desplazarse por los espacios que contempla el aula de forma libre y autónoma; c) acceso a las comunicaciones: los estudiantes deben poder recibir, emitir toda la información que circule en el aula para lo cual es necesario tener canales de tipo escrito, visual, auditivo, gestual y mixto.

\section{Método}

Para integrar en un solo modelo los aspectos antes identificados dentro del campo de la evaluación de actitudes hacia la educación inclusiva, el presente trabajo adopta el modelo teórico-metodológico de la teoría de facetas - propuesta por Louis Guttman (citado por Bilsky 2002, p. 4)-, la cual parte de la premisa de que en la investigación del comportamiento, que se ocupa generalmente con temas complejos, primero hay que conceptualizar y definir en términos sustantivos aquello que se está estudiando, y solo entonces se debe proceder a pruebas de diseño o cuestionarios para luego recopilar datos y, finalmente, realizar análisis estadísticos de los mismos.

A este respecto, Páramo (2008) señala:

lo que busca la teoría de facetas es la conceptualización a priori de un sistema de definiciones iniciales para guiar las observaciones que se quieran realizar sobre un campo particular, lo que supone la precisión de los límites de un área de investigación y la generación de una forma de expresión a tal definición de manera precisa (p. 85).
La teoría de la facetas contribuye al desarrollo de estudios para la elaboración de inventarios pues obliga al investigador a conceptualizar y definir sus construcciones de una manera formal y detallada. Adicionalmente el trabajo mediante la teoría de facetas permite el análisis multidimensional de datos que guiado por los conceptos definidos en las facetas permite conocer la estructura del inventario y contrastarla así con la frase mapa, la cual opera como hipótesis.

La faceta es un conjunto de atributos (variables) que en conjunto representan los componentes subyacentes, tanto conceptuales como semánticos, dentro de un universo de contenidos y que en su conjunto describen las variaciones dentro de una faceta que, para el caso del que se ocupa el presente estudio, se refieren a los componentes del campo de las actitudes hacia la educación inclusiva.

Las categorías de clasificación de cada observación dentro de las facetas son los elementos de las facetas. Los elementos se refieren a los diferentes valores que describen las variaciones de cada faceta. Se distinguen tres tipos de facetas:

Facetas de contexto: que se refieren a la población de sujetos considerados para el estudio. Facetas de contenido: que se refieren a las variables principales que los investigadores desean relacionar. Facetas de respuesta/(rango): que se refieren al universo de las reacciones o respuestas de los sujetos encuestados, también llamada rango de respuesta común. (Páramo, 2008, p. 86).

Las facetas son propuestas por el investigador, con el cuidado de que cada uno de los elementos que la componen deben ser mutuamente excluyentes y conceptualmente distintos unos de otros. Para el caso, las facetas identificadas fueron: la medida de las actitudes, los agentes y escenarios educativos, $y$ los elementos didácticos. Las facetas son condensadas en la frase mapa, columna vertebral de la teoría del modelo metodológico, la cual permite la selección equilibrada de las variables a ser medidas en el universo de contenido estudiado. En esta se establecen claramente cuáles son las facetas que componen del objeto a estudiar y se conceptualizan 
y desglosan las mismas. En esencia, la frase mapa ayuda a definir al fenómeno a estudiar teniendo en cuenta la población, las variables de contenido y el rango de posibles respuestas.

Las facetas trabajadas dentro de este proyecto derivan de las categorías enunciadas anteriormente: faceta de dominio A, las dimensiones de las actitudes (cognoscitivo, afectivo/emocional e incitadores a la acción/conductual); faceta de dominio B, los agentes y escenarios del proceso educativo: el docente, el estudiante, el aula y la institución educativa y faceta de dominio $\mathrm{C}$, los elementos didácticos: las estra- tegias, los recursos o materiales y los ambientes de aprendizaje. En cuanto a la faceta de rango común, esta se encuentra definida a partir del grado de acuerdo o desacuerdo del sujeto con las diferentes afirmaciones que se le plantean en relación a la educación inclusiva. Se emplea la siguiente escala como rango común: totalmente de acuerdo (TA), medianamente de acuerdo (MA), indiferente (I), medianamente en desacuerdo $(\mathrm{MD})$, totalmente en desacuerdo (TD). Estas facetas con los elementos que las componen se integraron en la frase mapa que se presenta en la tabla 1.

Tabla 1. Frase mapa para la identificación de las actitudes que tienen los docentes en formación hacia la educación inclusiva

\begin{tabular}{|c|c|}
\hline $\begin{array}{c}\text { Faceta de contexto } \\
\text { ¿En qué medida el estudiante de licencia- } \\
\text { tura }\end{array}$ & $\begin{array}{l}\text { Licenciatura } \\
\text { 1. Licenciatura en Educación con énfa- } \\
\text { sis en Educación Especial. } \\
\text { 2. Licenciatura en Educación Comunita- } \\
\text { ria con énfasis en Derechos Humanos. } \\
\text { 3. Licenciatura en Educación Infantil. } \\
\text { 4. Licenciatura en Psicología y Peda- } \\
\text { gogía. }\end{array}$ \\
\hline \multirow[t]{2}{*}{$\begin{array}{l}\text { Faceta de dominio A } \\
\text { Medida de la actitud } \\
\text { 1. Sabe o cree -cognoscitivos-. } \\
\text { 2. Tiene sentimientos, sensaciones o } \\
\text { preocupaciones -afectivos/emocionales- } \\
\text { 3. Cómo actúa o actuaría -incitadores a la } \\
\text { acción/conductuales- }\end{array}$} & \\
\hline & $\begin{array}{l}\quad \text { Faceta de dominio B } \\
\text { Agentes y escenarios educativos } \\
\text { Acerca de la incidencia que tiene la } \\
\text { educación inclusiva en los agentes y } \\
\text { escenarios educativos } \\
\text { 1) El docente. } \\
\text { 2) El estudiante. } \\
\text { 3) El aula. } \\
\text { 4) La institución educativa. }\end{array}$ \\
\hline \multirow[t]{2}{*}{$\begin{array}{l}\text { Faceta de dominio C } \\
\text { Elementos didácticos } \\
\text { Acerca de la incidencia que tiene el empleo } \\
\text { de elementos didácticos en la Educación } \\
\text { Inclusiva: } \\
\text { 1. Estrategias. } \\
\text { 2. Recursos o materiales. } \\
\text { 3. Ambientes de aprendizaje. }\end{array}$} & \\
\hline & \begin{tabular}{l}
\multicolumn{1}{c}{ Rango común } \\
1. Totalmente de acuerdo (TA). \\
2. Medianamente de acuerdo (MA). \\
3. Indiferente (I). \\
4. Medianamente en desacuerdo (MD). \\
5. Totalmente en desacuerdo (TD).
\end{tabular} \\
\hline
\end{tabular}


Habiendo definido las facetas y sus elementos, se establecen 18 perfiles a partir de la combinatoria de los mismos, a partir de los cuales se redactaron los ítems del instrumento con el que se evaluaron las actitudes de los maestros en formación que formaron parte de la investigación (anexo 1).

\section{Participantes}

Para la selección de la muestra se optó por un muestreo no probabilístico en el cual se tuvieron en cuenta las cuatro licenciaturas de la Facultad de Educación. Posteriormente, se buscó el balance de la muestra en relación a los programas académicos seleccionados: Licenciatura en Educación con énfasis en Educación Especial, Licenciatura en Educación Infantil, Licenciatura en Psicología y Pedagogía y Licenciatura en Educación Comunitaria con énfasis en Derechos Humanos.

Igualar las muestras en relación a los programas académicos permite establecer comparaciones y descripciones relacionadas con las particularidades de formación que oferta cada uno de los planes de estudio derivados de los campos de acción específica de cada programa. Adicionalmente este ejercicio facilita la generalización de los datos referidos al instrumento y derivados de este en relación con los estudiantes de licenciaturas en educación de la UPN. La muestra participante de este estudio se distribuye como se indica en la tabla 2.

Tabla 2. Distribución de los participantes

\begin{tabular}{l|c|c}
\multicolumn{1}{c|}{ Licenciatura } & Total & $\%$ \\
\hline $\begin{array}{l}\text { Licenciatura en Educación con } \\
\text { énfasis en Educación Especial }\end{array}$ & 24 & $27 \%$ \\
\hline $\begin{array}{l}\text { Licenciatura en Educación In- } \\
\text { fantil }\end{array}$ & 21 & $23 \%$ \\
\hline $\begin{array}{l}\text { Licenciatura en Educación Co- } \\
\text { munitaria con énfasis en Dere- } \\
\text { chos Humanos }\end{array}$ & 22 & $24 \%$ \\
\hline $\begin{array}{l}\text { Licenciatura en Psicología y } \\
\text { Pedagogía }\end{array}$ & 24 & $26 \%$ \\
\hline \multicolumn{1}{c|}{ Total } & 91 & $100 \%$ \\
\hline
\end{tabular}

\section{Procedimiento}

Siguiendo el procedimiento establecido para la elaboración de cuestionarios a través de la teoría de facetas (Páramo, 2008), se generaron dos ítems por cada una de las combinaciones derivadas de las facetas de dominio de la investigación (A-B, A-C). Una vez definidas las facetas y diseñado el cuestionario a partir de la frase mapa, estos ítems fueron sometidos a una validación de contenido por parte de un panel de tres expertos quienes valoraron los ítems. Para la evaluación de los ítems se tuvieron en cuenta cuatro criterios: a) la definición precisa del dominio, b) el grado de suficiencia con que ese dominio se evalúa, c) relevancia del ítem y d) claridad y calidad de la redacción del ítem.

Teniendo en cuenta que la teoría de facetas es una estrategia de investigación que integra el análisis formal del contenido de la investigación, con el análisis de datos multivariado como lo es el SSA (Smallest Space Analysis), este modelo metodológico permite una reflexión conjunta integrando el diseño de contenidos y el análisis de datos los cuales se discuten de forma interactiva para aportar a la construcción de conocimiento y la medición significativa de aspectos específicos de la conducta, en este caso las actitudes hacia la educación inclusiva.

Para realizar este proceso se realizó un pilotaje del instrumento con 79 estudiantes de las licenciaturas en Educación con énfasis en Educación Especial y en Educación Infantil del convenio Universidad Pedagógica Nacional - Normal Superior Santiago de Cali.

Una vez realizada la aplicación del instrumento en su versión inicial, se revisó la estructura del mismo a partir del análisis de espacio más pequeño (SSA) empleando el software Lifa2000. A partir de este proceso se ajustó el instrumento teniendo además en cuenta las observaciones en cuanto a redacción y diseño del cuestionario realizadas por los estudiantes participantes en el pilotaje. Finalmente, el instrumento fue aplicado a una muestra de 91 estudiantes de las licenciaturas de la Facultad de Educación de la UPN. 


\section{Resultados}

Estructura del inventario. A partir de los datos surgidos de la aplicación se llevó a cabo el análisis de espacio más pequeño (SSA) empleando el software Lifa2000 para conocer la estructura fundamental del inventario y validar sus resultados a partir de las facetas definidas.

Teniendo en cuenta la frase mapa se analiza inicialmente cada uno de los gráficos que arroja el programa para cada una de las facetas. En este análisis se tiene en cuenta la ubicación de los ítems en el espacio y sus diferentes agrupaciones de manera que si los ítems que teóricamente forman parte de un elemento de la faceta se pueden agrupar en una misma región, entonces se mostrará evidencia de la faceta propuesta. Este análisis permite observar si la estructura interna del cuestionario corresponde con las facetas definidas en la frase mapa. El análisis de espacio más pequeño (SSA) lleva a que el investigador trate de darle sentido a la cercanía espacial entre los ítems, respondiendo a una categoría conceptual que explique la agrupación y que deberá responder en principio, a los elementos que se han propuesto en la faceta que se desea estudiar. De no coincidir exactamente con ellos, deberá proponer algún otro concepto que permita entender la agrupación de los ítems. En caso de que las agrupaciones de los ítems no respondan a ninguno de los elementos que componen la faceta, esta deberá descartarse como variable que explique los resultados.

\section{Faceta actitudes (A)}

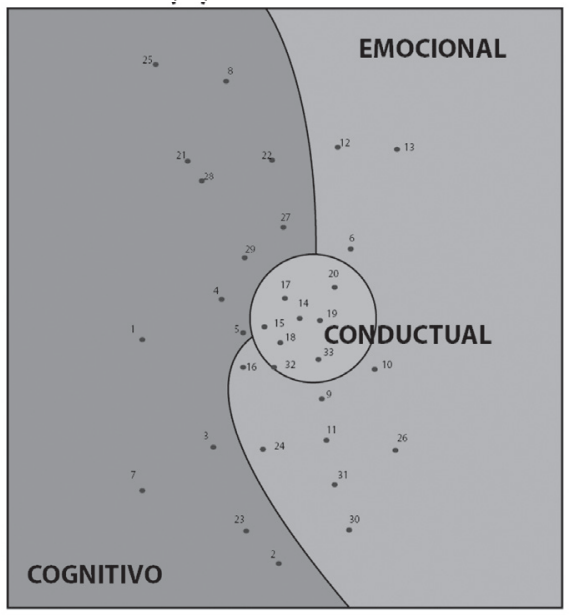

Figura. 1. Gráfico SSA de la faceta actitudes (A)
En el instrumento se define la faceta actitud teniendo en cuenta los componentes: a) cognoscitivos: saberes o creencias, b) afectivos/emocionales: sentimientos, sensaciones o preocupaciones, c) incitadores a la acción/conductuales: actuaciones presentes o futuras.

Como puede observarse en la figura 1 , los ítems que conforman el componente cognoscitivo están ubicados en la región lateral izquierda de la gráfica y se muestran de modo axial. Se incluyen en esta región los ítems 1, 2, 3, 4, 5, 7, 8, 23, 25, 27 y 28. Se excluye de esta región únicamente el ítem 6: "Es más difícil el manejo de la disciplina en un aula inclusiva que en un aula regional/tradicional", este se ubica dentro del componente afectivo/emocional, lo que podría indicar que los estudiantes relacionan esta afirmación desde sus emociones, sensaciones o preocupaciones más que como un elemento cognoscitivo.

Adicionalmente se observa que se agrupan en esta región los ítems 21: "Las escuelas deberían tener aulas especializadas de acuerdo con las particularidades de los estudiantes" y 22: "Debería haber escuelas inclusivas y escuelas regulares/tradicionales". Estos se definieron inicialmente en el componente incitadores a la acción/conductual, sin embargo parecen responder más al componente cognoscitivo, siendo interpretadas las afirmaciones por parte de los participantes como conocimientos o creencias en relación a la educación inclusiva.

Se observa igualmente que el componente afectivo/emocional queda ubicado en la región lateral derecha de la gráfica y se muestra de modo axial. En esta región quedan incluidos los ítems 9, $10,11,12,13,16,24,26$ y 30 . Se encuentra que el ítem 31: "Como docente de aula inclusiva seguiría empleando las mismas estrategias de enseñanza que conozco" que inicialmente fue planteado en el componente cognoscitivo se agrupa dentro del afectivo/emocional y se observa su relación con los ítems de las emociones, preocupaciones y sentimientos. El ítem 6 se agrupa a este componente en la gráfica como se había descrito anteriormente. 
Por último, el componente incitadores a la acción/conductual queda ubicado en la región media de la gráfica de forma concéntrica/modular, lo que indica que sobre estos aspectos, todos los participantes estuvieron de acuerdo dado el grado de correlación interna entre los ítems, mostrando así, un grado de consenso entre todos los participantes sobre este elemento de la actitud 5 . Esta región queda conformada por los ítems 14, 15, 17, 18, 19, 20, 32 y 33. El ítem 14, "Me preocupa que todas las aulas se transformen en inclusivas", inicialmente se definió en el componente afectivo/emocional y después de ser analizados los datos quedó ubicado en el centro de la gráfica como parte del componente incitadores a la acción/conductual. Los ítems restantes (15, $17,18,19,20,32$ y 33) habían sido inicialmente planteados en este componente. Quedan por fuera de esta región los ítems 21 y 22 agrupándose en el componente cognoscitivo; por su parte el ítem 31 queda ubicado en el afectivo/emocional como anteriormente se había descrito.

A partir de la distribución de estos datos se da evidencia de la faceta actitud con sus tres componentes claramente definidos: el cognoscitivo y el afectivo/emocional se muestran agrupados de modo axial de tal manera que no expresan relaciones entre ellos, es decir los sentimientos, emociones y preocupaciones no dependen o se relacionan con las creencias y pensamientos, es decir, se muestran como elementos independientes. Por el contrario, la faceta incitadores a la acción/conductual se muestra de modo modular y se ubica en el centro del plano entre los componentes cognoscitivo y afectivo/ emocional lo que demuestra la relación de estos dos componentes con el incitadores a la acción/conductual; es decir, las acciones que realiza el estudiante o que estaría dispuesto a realizar dependen de sus sentimientos, preocupaciones, sentimientos, creencias y pensamientos en torno a la educación inclusiva.

5 Para ampliar información respecto al análisis de espacio más pequeño (SSA), revisar Bilsky (2002).

\section{Faceta agentes y escenarios educativos (B)}

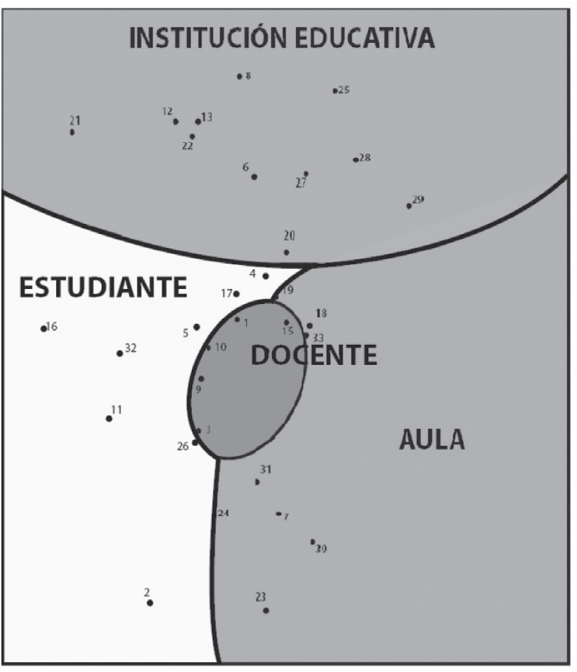

Figura 2. Gráfico SSA de la faceta agentes y escenarios educativos (B)

Esta faceta se propuso con cuatro agentes: el docente, el estudiante, el aula, la institución educativa. Se observa en el gráfico que arroja el programa Lifa2000 que el componente docente queda ubicado en la región central entre los componentes estudiante y aula mostrándose de modo concéntrico modular.

En la región central se ubican los ítems 1, 3, 9, 10,15 , los cuales inicialmente habían sido planteados para el componente docente. A continuación se realiza el análisis de cada uno de los componentes de la faceta.

Se registra que el componente estudiante queda ubicado en la región lateral inferior izquierda de la gráfica y se muestra de modo axial. En esta región se incluyen los ítems $2,4,5,16$ y 17 . Todos los ítems agrupados en esta región habían sido planteados en este componente. Por otra parte, el ítem 11, "Me preocupa que en un aula inclusiva no alcance a dar todos los contenidos del plan de estudios", y el ítem 14, "Me preocupa que todas las aulas se transformen en inclusivas", fueron planteados inicialmente en el componente aula, sin embargo, correlacionan con los ítems del componente estudiante. Podría decirse que los sujetos participantes interpretan estos dos ítems desde la preocupación hacia la afección directa o indirecta a los estudiantes participantes de la educación inclusiva. 
La región para el componente aula queda ubicada en la parte inferior lateral derecha de la gráfica mostrándose de modo axial. En esta región se incluyen los ítems 7,18 y 19 . Todos los ítems antes mencionados habían sido definidos inicialmente en este componente. Se excluye de esta región el ítem 6: "Es más difícil el manejo de la disciplina en un aula inclusiva que en un aula regional/tradicional", el cual se ubica en el componente institución educativa. También quedan excluidos los ítems 11 y 14, los cuales se incluyen en el componente estudiante siendo analizados anteriormente.

Se observa que el componente institución educativa queda ubicado en la región superior de la gráfica de forma axial. Esta región está conformada por los ítems 8, 12, 13, 20, 21 y 22, los cuales habían sido planteados en el componente institución educativa a excepción del ítem 6, "Es más difícil el manejo de la disciplina en un aula inclusiva que en un aula regional/tradicional", el cual inicialmente se definió en el componente aula y en este caso los participantes del estudio parecen percibir la disciplina como un asunto de orden institucional.

A partir de los datos se evidencia que la faceta agentes y escenarios educativos tiene cuatro componentes definidos. Se advierte que el componente institución presenta un modo axial en la región superior siendo perpendicular a los componentes estudiante y aula. Finalmente el componente docente parece ser el más fuerte ubicándose de modo concéntrico/modular en el centro de la gráfica y mostrándose como eje conector entre el estudiante y el aula; lo anterior se muestra en contraste con la ubicación de la institución, lo que pareciera indicar que los efectos de la educación inclusiva se dan de forma aislada a este agente o contexto educativo o que el docente percibe alejadas las políticas de la institución a su labor directa, al estudiante y al aula.

\section{Faceta elementos didácticos (C)}

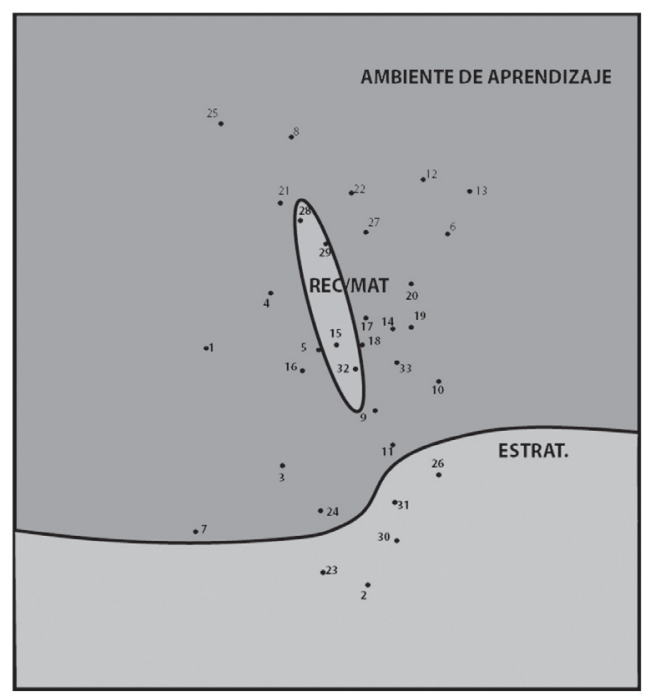

Figura 3. Gráfico SSA de la faceta elementos didácticos (C)

Se observa que el componente estrategias se ubica en la región inferior derecha y se muestra de modo axial. En esta región se encuentran los ítems 23, 26, 30 y 31 . Se excluye de esta región el ítem 27, "Estoy convencido que el empleo de estrategias inclusivas mejora el clima del aula", el cual se ubica con los ítems del componente ambientes de aprendizaje.

Se aprecia que el componente recursos o materiales está en la región central de la gráfica mostrándose de modo concéntrico/modular. Quedan incluidos en esta región los ítems 28, 29 y 32 . Esta región queda constituida únicamente por los ítems inicialmente planteados para el componente recursos $o$ materiales.

Es posible identificar que el componente ambientes de aprendizaje se ubica en la región superior de la gráfica y se muestra de modo axial. En esta región se encuentran los ítems 24, 25 y 33 . Los ítems que integran esta región habían sido definidos inicialmente en este componente y se caracterizan por haber quedado dispersos en el espacio. Adicionalmente, el ítem 27, "Estoy convencido que el empleo de estrategias inclusivas mejora el clima del aula”, se agrega a la región pese a haber sido definido inicialmente en el componente estrategias. La presencia de este ítem en este componente puede deberse a que los 
participantes identifican el clima del aula más en relación con ambientes de aprendizaje que con las estrategias.

El componente recursos o materiales está en la región central de la gráfica, quedando rodeado por los ambientes de aprendizaje, lo que muestra una relación jerárquica entre estos dos componentes. Por su parte, el componente estrategias queda aislado en la parte inferior derecha de la gráfica, lo que indica independencia de los otros dos. Lo anterior muestra que, para los participantes del estudio, los recursos o materiales son fundamentales para el desarrollo de los ambientes de aprendizaje y, a su vez, se constituyen en el eje de la educación inclusiva; por su parte las estrategias muestran una alta correlación entre sus elementos manteniendo su independencia, es decir, no dependen de los otros dos componentes.

\section{Análisis de relación entre las facetas}

Al realizar un análisis conjunto de las tres facetas se encuentra que los participantes del estudio identifican como eje de la educación inclusiva al docente y sus acciones, y los recursos o materiales con los que este cuenta para su ejercicio; esto debido a que ambos elementos quedan ubicados en la zona central al realizar la conjunción de ambas gráficas. Se observa entonces que estos tres elementos se entretejen como núcleo del proceso inclusivo y son considerados por los participantes como los más importantes de acuerdo con la ubicación de estos en cada una de las gráficas.

Llama la atención que el componente estrategias, en la gráfica de la faceta $\mathrm{C}$, se encuentra ubicado en la parte inferior de esta sin ocupar un lugar importante y presentándose aislado. Sin embargo, al trasponer las tres facetas se entrecruza con el componente aula y distanciado de los componentes institución y docente. Lo anterior permite presumir que los sujetos participantes del estudio están percibiendo las estrategias como elementos particulares de cada aula sin responder a un contexto institucional o a un docente particular. A partir de esto se evidencia la necesidad de incluir este componente en la formación de los futuros licenciados pues aunque las estrategias se sitúan directamente en el contexto del aula, estas se diseñan y se aplican dentro de un marco institucional particular y son adecuadas y moldeadas por las individualidades de los docentes, quienes las hacen propias en la ejecución diaria.

Es claro que de la faceta agentes y escenarios educativos, el docente es el más importante en la reflexión que los participantes hacen, es decir, ellos consideran que la mayor responsabilidad de este proceso recae en los docentes y que la educación inclusiva se debe dar a partir de acciones las cuales son el resultado de sus creencias y sus sentimientos.

Por otro lado vemos cómo el componente recursos o materiales cobra importancia en el proceso, es decir, los participantes perciben que sí se requiere una serie de recursos o materiales para que el docente lleve a cabo las prácticas inclusivas y que no es posible lograr la inclusión si no se cuenta con estos. Al trasponer las gráficas se aprecia que el componente de recursos o materiales se entrelaza en una parte con el componente institución y en otra con el componente docente, lo que lleva a pensar que los participantes consideran que los esfuerzos para la consecución de material deben ser dados por la institución educativa, siendo su empleo responsabilidad del docente.

El componente institución cobra mayor fuerza al trasponer las gráficas pues se ubica en la región superior de estas, segmentando de forma proporcional los componentes cognoscitivo, afectivo/ emocional, incitadores a la acción/conductual, recursos o materiales y ambientes de aprendizaje. Lo anterior muestra que para los participantes del estudio, la institución, como contexto y como espacio institucional que demarca los procesos de enseñanza-aprendizaje que se dan al interior de la misma, tiene una influencia grande en los procesos de educación inclusiva ya sea con la determinación de ser incluyente, así como con la consecución de recursos apropiados para la atención o la capacitación a los docentes en aras de cualificar sus prácticas inclusivas. 


\section{Análisis estadístico descriptivo}

En este apartado se realiza un análisis descriptivo de las respuestas dadas por los estudiantes de licenciatura que participaron en el estudio. También se presentan las medias obtenidas de cada una de las facetas definidas por cada licenciatura. Para efectos de dicho análisis, la escala de aquellos ítems que están formulados en dirección opuesta a la inclusión, fue invertida.

Tabla 3. Medias y desviaciones típicas de los componentes por programa

\begin{tabular}{|c|c|c|c|c|c|c|c|c|}
\hline & \multicolumn{8}{|c|}{ PROGRAMA ACADÉMICO } \\
\hline & \multicolumn{2}{|c|}{$\begin{array}{c}\text { Licenciatura en Educa- } \\
\text { ción Comunitaria con } \\
\text { Énfasis en Derechos } \\
\text { Humanos }\end{array}$} & \multicolumn{2}{|c|}{$\begin{array}{l}\text { Licenciatura en } \\
\text { Educación con Én- } \\
\text { fasis en Educación } \\
\text { Especial }\end{array}$} & \multicolumn{2}{|c|}{$\begin{array}{l}\text { Licenciatura en Edu- } \\
\text { cación Infantil }\end{array}$} & \multicolumn{2}{|c|}{$\begin{array}{l}\text { Licenciatura en Psi- } \\
\text { cología y Pedagogía }\end{array}$} \\
\hline & Media & $\begin{array}{l}\text { Desviación } \\
\text { típica }\end{array}$ & Media & $\begin{array}{l}\text { Desviación } \\
\text { típica }\end{array}$ & Media & $\begin{array}{l}\text { Desviación } \\
\text { típica }\end{array}$ & Media & $\begin{array}{l}\text { Desviación } \\
\text { típica }\end{array}$ \\
\hline TOTAL & 4,23 & 00,51 & 4,27 & 0,42 & 4,23 & 0,43 & 4,26 & 0,41 \\
\hline Cognoscitivo & 4,20 & 0,45 & 3,94 & 0,57 & 3,90 & 0,45 & 4,00 & 0,47 \\
\hline Afectivo/Emocional & 3,10 & 0,68 & 3,34 & 0,64 & 3,46 & 0,59 & 3,40 & 0,62 \\
\hline $\begin{array}{c}\text { Incitadores a la acción/ } \\
\text { Conductual }\end{array}$ & 3,95 & 0,67 & 4,14 & 0,56 & 3,92 & 0,63 & 3,97 & 0,47 \\
\hline Docente & 4,35 & 0,71 & 4,23 & 0,54 & 3,96 & 0,72 & 4,30 & 0,67 \\
\hline Estudiante & 3,75 & 0,79 & 3,90 & 0,71 & 3,69 & 0,57 & 3,53 & 0,60 \\
\hline Aula & 3,73 & 0,61 & 3,51 & 0,62 & 3,80 & 0,65 & 3,68 & 0,59 \\
\hline Institución & 2,96 & 0,67 & 3,25 & 0,85 & 3,47 & 0,59 & 3,28 & 0,80 \\
\hline Estrategias & 3,73 & 1,04 & 3,84 & 0,80 & 3,74 & 0,73 & 3,99 & 0,69 \\
\hline Recursos o materiales & 3,76 & 0,93 & 3,85 & 0,65 & 3,94 & 0,73 & 3,85 & 0,67 \\
\hline Ambientes de aprendizaje & 4,16 & 0,74 & 4,33 & 0,72 & 3,98 & 0,86 & 4,36 & 0,51 \\
\hline
\end{tabular}

Se puede observar que en general las medias son altas, siendo la más baja la obtenida en el componente institución $(2,96)$ por los estudiantes de la Licenciatura en Educación Comunitaria con Énfasis en Derechos Humanos. Por su parte, la media más alta la obtiene en el componente ambientes de aprendizaje la Licenciatura en Psicología y Pedagogía.

Se destaca que en el puntaje total obtenido en la prueba, los puntajes son muy similares sin mostrar diferencias estadísticamente significativas, siendo la Licenciatura en Educación con Énfasis en Educación Especial la que obtiene el puntaje más alto $(4,27)$.

En la tabla 4 se muestran las medias y desviaciones estándar por ítem. A partir de estos datos se realiza un análisis descriptivo que permite observar el comportamiento tanto del instrumento como de los participantes del estudio en relación a sus actitudes hacia la educación inclusiva. 
Tabla 4. Medias y desviaciones típicas por ítem

\begin{tabular}{|c|c|c|c|c|c|}
\hline İtem & Media & $\begin{array}{c}\text { Desv. } \\
\text { tip. }\end{array}$ & ítem & Media & Desv. tip. \\
\hline 1 & 4,71 & 0,67 & 18 & 2,80 & 1,59 \\
\hline 2 & 4,20 & 1,19 & 18 & 2,62 & 1,60 \\
\hline 3 & 3,96 & 1,38 & 20 & 3,27 & 1,36 \\
\hline 4 & 4,27 & 0,94 & 21 & 4,11 & 1,25 \\
\hline 5 & 4,29 & 1,12 & 22 & 3,70 & 1,45 \\
\hline 6 & 3,17 & 1,43 & 23 & 3,98 & 1,42 \\
7 & 3,19 & 1,23 & 24 & 4,56 & 0,88 \\
\hline 8 & 4,00 & 1,40 & 25 & 4,22 & 1,14 \\
\hline 9 & 3,51 & 1,53 & 26 & 4,34 & 1,09 \\
10 & 4,31 & 1,02 & 27 & 4,67 & 0,79 \\
11 & 4,53 & 0,94 & 28 & 4,61 & 0,79 \\
\hline 12 & 3,98 & 1,33 & 29 & 2,09 & 1,24 \\
\hline 13 & 4,23 & 1,11 & 30 & 3,33 & 1,49 \\
14 & 3,60 & 1,39 & 31 & 4,04 & 1,28 \\
15 & 1,86 & 1,16 & 32 & 3,35 & 1,42 \\
16 & 2,97 & 1,47 & 33 & 4,69 & 0,84 \\
17 & 3,51 & 1,54 & & & \\
\hline & & & & & \\
\hline
\end{tabular}

Los datos arrojados por el análisis descriptivo de los ítems muestran que en general se presentan puntajes altos. Llama la atención que 17 de los 33 ítems registran medias superiores a 4,00 siendo el ítem 29, "Las escuelas deberían tener aulas especializadas de acuerdo a las particularidades de los estudiantes", el que obtiene la media más baja $(\bar{x}=2,09, \sigma$ $=1,24)$. Se observa que los estudiantes tienen un nivel moderado de acuerdo con la afirmación y una dispersión de 1,24 entre sus respuestas, lo que indica que pese a que los estudiantes no muestran un alto acuerdo, sus respuestas tienden al grado acuerdo. Esto indica que, en contraposición a los postulados de la educación inclusiva, ellos consideran que se deberían mantener las aulas especializadas para atender a los estudiantes con particularidades diferentes al promedio, conservando aun la idea de mantener las modalidades educativas como una estrategia institucional de atención a la diversidad de la población estudiantil.

Por su parte, el ítem 33, "Estaría dispuesto a trabajar por proyectos de aula, rincones de aprendizaje u otra organización flexible que favorezca el aprendizaje y participación de todos los estudiantes", obtiene la media más alta $(\bar{x}=4,69, \sigma=1,42)$. Teniendo en cuenta que la escala de este ítem fue invertida, los datos nos muestran que los estudiantes tienen un nivel alto de acuerdo con la afirmación y estarían en disposición de ejecutar acciones relacionadas a flexibilizar y enriquecer los ambientes de aprendizaje para favorecer los procesos de los estudiantes. Pese a que este ítem muestra una alta dispersión de los datos, sigue mostrando una alta disposición de los estudiantes hacia el ejercicio de las acciones en relación al empleo de organizaciones flexibles en el aula.

En general, los datos muestran un alto nivel de acuerdo entre los estudiantes, siendo el ítem 18, "Me inquieta que la educación inclusiva sea una política educativa obligatoria", el que presenta la desviación más alta $(\sigma=1,60, \bar{x}=2,80)$. Por su parte, el ítem 1 , "El docente de un aula inclusiva requiere conocer estrategias pedagógicas diferentes", presenta la desviación más baja $(\sigma=0,67, \bar{x}=4,71)$

Lo anterior muestra que los estudiantes tienden a tener actitudes favorables hacia la educación inclusiva.

\section{Conclusiones}

El objetivo de este estudio fue la construcción y validación de un instrumento para medir actitudes hacia la educación inclusiva basado en el modelo de investigación de teoría de facetas; se puede concluir, a partir de los resultados del análisis de espacio más pequeño (SSA) que existe evidencia empírica para afirmar que las facetas se estructuran como estaban inicialmente propuestas en la frase mapa.

Se evidencian tres facetas que en conjunto describen las actitudes de los estudiantes de las licenciaturas de la Facultad de Educación hacia la educación inclusiva: actitudes, agentes y contextos educativos, y elementos didácticos.

La faceta actitud muestra que los componentes cognoscitivo y afectivo/emocional se agrupan de modo axial, lo que permite afirmar que las relaciones entre ellos son diferenciables. Por otro lado, la faceta incitadores a la acción/conductual se muestra de modo modular ubicándose en el centro del plano entre los componentes cognoscitivo y afectivo/ emocional lo que demuestra la relación de estos 
dos componentes con el incitadores a la acción/ conductual. Lo anterior muestra que las acciones que realiza el estudiante o que estaría dispuesto a realizar, dependen del componente afectivo/emocional y cognoscitivo. Es importante pensar que los programas de formación deben preocuparse más por buscar un mayor nivel de coherencia entre los conocimientos y contenidos sobre el tema de educación inclusiva y la relación con lo que el docente puede y debe hacer, es decir, su rol como docente en un contexto inclusivo.

En cuanto a la faceta agentes y escenarios educativos se resalta que el componente docente parece ser el más fuerte ubicándose de modo concéntrico/ modular en el centro de la gráfica y mostrándose como eje conector entre el estudiante y el aula. Por otra parte, se observa que es necesario trabajar sobre los elementos relacionados con el aula, pues los ítems a que se refiere este componente se ubican de forma dispersa en la gráfica; solo la mitad de ellos se agrupan, por lo que convendría trabajar sobre la importancia que tienen los elementos del aula para efectos del trabajo didáctico en la clase.

Además, los estudiantes no ven interdependencia entre lo que ocurre en el aula y aquello que ocurre en la institución, esto debido a que las dos regiones quedan apartadas y no se ven relacionadas, pudiéndose interpretar como factores aislados. Estos dos componentes parecieran quedar desligados.

En la faceta elementos didácticos se observa que el componente recursos o materiales es visto como el eje central del proceso inclusivo. En segundo nivel de importancia se ubica el componente ambientes de aprendizaje. Por último se visualiza el componente Estrategias el cual se encuentra distante de los otros dos, lo cual supone independencia. Llama la atención que los ítems del componente de ambientes de aprendizaje quedan muy dispersos, por lo que es necesario revisar el concepto que tejen alrededor de este. Por otra parte, se requiere pensar qué elementos de la formación están generando que se perciban los recursos o materiales como un elemento didáctico indispensable para el éxito de la educación inclusiva, cuando son las estrategias las que realmente deberían ocupar el lugar central.
Es fundamental reconocer que los docentes en formación son seres integrales con una historia de vida y unas experiencias previas que se ven reflejadas en sus sentimientos, creencias y en sus comportamientos. Por supuesto, el reconocer la integralidad del ser humano y la interrelación entre las creencias, los sentimientos y los aspectos instrumentales como constituyentes del comportamiento de las personas, lleva a reflexionar sobre los procesos de formación que actualmente se están dando dentro de las aulas formadoras de docentes. Claramente los estudios descritos por Barón y Byrne (1998), han mostrado que los cambios actitudinales no se dan únicamente a partir de la información y el conocimiento, lo cual es el eje fundamental de los planes de formación de las licenciaturas. Es necesario profundizar en los aspectos académicos de las licenciaturas estudiadas, ya que no se encuentran diferencias estadísticamente significativas que permitan afirmar que la actitud hacia la educación inclusiva de los docentes en formación de determinada licenciatura (faceta de contexto) es más positiva que de los docentes en formación de otra. Lo anterior presenta la necesidad de realizar una reflexión y análisis acerca del papel de la formación en las actitudes.

Paralelamente es necesario trabajar de forma intencional el reconocimiento de los sentimientos, los miedos y las creencias que el estudiante trae consigo y que derivan de su formación en diferentes contextos previos a su ingreso a las licenciaturas.

Dada la importancia que tienen las actitudes hacia la educación inclusiva en el comportamiento de los futuros licenciados frente a la misma, resulta fundamental plantear procesos de formación específica que se den a partir del reconocimiento de las actitudes inicialmente hacia la diferencia y hacia la diversidad para finalizar en su desarrollo en los contextos educativos inclusivos. En este orden de ideas, es fundamental tomar en cuenta, reconocer e involucrar dentro de la formación las historias de vida de los estudiantes, las concepciones que sobre la diferencia se teje al interior de las familias, las experiencias educativas y otros elementos de la socialización personal. 
Ahora bien, se deben reconocer las actitudes de los estudiantes hacia la Educación Inclusiva ya que estos son los futuros docentes que la pondrán en práctica. Este estudio muestra cómo los docentes en formación están percibiendo la educación inclusiva, reto del cual deben dar cuenta en sus prácticas docentes. Los resultados hacen pensar que los futuros licenciados al egresar tendrán una buena disposición hacia el trabajo en contextos inclusivos. Sin embargo esta disposición que muestran no puede capitalizarse de no contar con las estrategias y herramientas necesarias para llevar a cabo su ejecución de forma exitosa.

Se hace necesario en consecuencia fortalecer la formación en tres ejes fundamentales: inicialmente reconociendo y trabajando sobre las actitudes de los docentes en formación; en segundo lugar brindándoles los conocimientos, las estrategias y herramientas necesarias para lograr realizar una intervención inclusiva adecuada; y tercero, disponer de espacios de práctica donde el docente en formación pueda prepararse para generar procesos inclusivos exitosos, teniendo en cuenta que la experiencia didáctica ejerce una fuerte influencia sobre la actitud y la conducta.

Esta triada en la formación plantea un abordaje integral, pues una disposición favorable no es suficiente para enfrentar la realidad de un aula inclusiva y de no contar con los elementos de intervención necesarios puede llegarse fácilmente a experiencias no exitosas que pueden modificar esta disposición rápidamente.

A partir de los resultados de esta investigación queda abierta la posibilidad de realizar futuros estudios que en esta dirección amplíen la visión e información que se tiene en relación a la medición de actitudes hacia la educación inclusiva. Es conveniente, por tanto, explorar en futuras investigaciones, nuevas facetas a la frase mapa que aquí ha servido para indagar por las actitudes, como lo pueden ser las diferentes poblaciones atendidas en procesos de educación inclusiva, o incluir en la faceta agentes educativos, otros elementos que integren diversos actores que en este estudio no fueron contemplados como son, por ejemplo, la familia y los directivos docentes.

\section{Referencias bibliográficas}

Aguilar, A. (2010). Impacto de la actitud docente en la vida académica de sus alumnos. Recuperado el 7 de octubre de 2012 de: http://www.upn081.edu.mx/ EIE2010/CCHEP/SUJETOS\%20DE\% 20LA\%20 EDUCACION/1ATA.pdf

Alegre de la Rosa, O. M. (2000). Diversidad humana y educación. Málaga: Aljibe.

Arnáiz, P. (2003). Educación inclusiva: una escuela para todos. España: Aljibe.

Barón, R.A. y Byrne, D. (1998). Psicología social. Madrid: Prentice Hall.

Bilsky W, (2002). La teoría de facetas: Investigaciones Básicas y Aplicaciones Paradigmáticas. Recuperado el 5 de septiembre de 2012 de: http://wwwpsy.uni-muenster. $\mathrm{de} /$ imperia/md/content/psychologie_institut_4/ ae_bilsky/forschungsberichte_ae/fb_25.pdf

Cabero, J. (2001). Tecnología educativa, diseño y utilización de medios para la enseñanza. Madrid: Paidós.

Ministerio de Educación Nacional (2008). Cartilla de Educación Inclusiva. Guía y herramienta: educación inclusiva con calidad. "Construyendo capacidad institucional para la atención a la diversidad". Serie de Guías No. 34, Bogotá.

Páramo P. (2008). La investigación en ciencias sociales. Bogotá: Universidad Piloto de Colombia.

Fishbein, M., y Ajzen, I. (1975). Belief, Attitude, Intention, and Behavior: An Introduction to Theory and Research. Recuperado el 25 de noviembre de 2011 de: http://people.umass.edu/aizen/f\&a1975.html

Sales, A.; Moliner, O. y Sanchiz, M. (2001). Actitudes hacia la atención a la diversidad en la formación inicial del profesorado. Revista Electrónica Interuniversitaria de Formación del Profesorado, 4(2).

Sánchez, A., Díaz C., Sanhueza S., Carrillo, M. (2008). Percepciones y Actitudes de los Estudiantes de Pedagogía hacia la Inclusión Educativa. En Estudios Pedagógicos, 34 (2). Valdivia-Chile. Recuperado de http://www.scielo.cl/pdf/estped/v34n2/art10.pdf

Sola, T. (1999). La formación inicial y su incidencia en la educación especial. En A. Sánchez y J.A. Torres. Educación Especial I. Una perspectiva curricular, organizativa y profesional. Madrid: Pirámide. p.p. 389-406

Stainback, S., \& y Stainback, W. (1999). Aulas inclusivas. Un solo sistema una única finalidad. Baltimore: Paul Brooks.

\footnotetext{
FOLIOS n. ${ }^{\circ} 37$
} 
Tilstone, C.; Florian, L. y Rose, R. (2003). Promoción y desarrollo de prácticas educativas inclusivas. Madrid: EOS.

Unesco. (2009). Directrices sobre políticas de inclusión en la educación. París. Recuperado el 5 de junio de 2011 de http://unesdoc.unesco.org/ images/0017/001778/177849s.pdf

Vélez, L. (2011). La educación inclusiva en los programas de formación inicial de docentes. Proyecto de investigación doctoral. Doctorado Interinstitucional en Educación. Bogotá: Universidad Pedagógica Nacional.

\section{Anexo 1. Instrumento}

\section{Actitudes hacia la educación inclusiva en docentes en formación}

..Mi nombre es Libia Vélez Latorre, soy estudiante del Doctorado Interinstitucional en Educación de la Universidad Pedagógica Nacional y actualmente adelanto un estudio en relación a la educación inclusiva. El presente instrumento tiene como propósito identificar sus actitudes sobre la educación inclusiva. Se entiende la educación inclusiva como la posibilidad de acoger en la institución educativa a todos los estudiantes valorando la diversidad de sus características personales y culturales.

Solicito su colaboración para el diligenciamiento de este cuestionario como docente en formación de la UPN. Sus respuestas son anónimas. Los resultados derivados de este instrumento serán usados únicamente con propósitos de investigación y no tendrán incidencia alguna en su proceso académico. Si desean hacer algún aporte al instrumento o tienen alguna duda sobre este pueden escribirme al correo electrónico libiavelezlatorre@yahoo.com.ar

Por favor complete los siguientes datos de identificación: Género: Edad:

Licenciatura: Semestre académico que cursa:

Fecha del diligenciamiento:

A continuación encontrará una serie de afirmaciones las cuales debe leer cuidadosamente. Marque con una X la casilla correspondiente al nivel de acuerdo o desacuerdo con las afirmaciones enunciadas sobre la educación inclusiva, teniendo en cuenta las siguientes opciones:

- Totalmente de acuerdo (TA)

- Medianamente de acuerdo (MA)

- Indiferente (I)

- Medianamente en desacuerdo (MD)

- Totalmente en desacuerdo (TD) 
Universidad Pedagógica Nacional

Facultad de Humanidades

\begin{tabular}{|c|c|c|c|c|c|c|c|}
\hline & & & $\begin{array}{c}\text { Total } \\
\text { acuerdo }\end{array}$ & $\begin{array}{l}\text { Moderado } \\
\text { acuerdo }\end{array}$ & Indiferente & $\begin{array}{l}\text { Moderado } \\
\text { desacuerdo }\end{array}$ & $\begin{array}{c}\text { Total } \\
\text { desacuerdo }\end{array}$ \\
\hline 1 & $\begin{array}{l}\mathrm{A} 1 \\
\mathrm{~B} 1\end{array}$ & $\begin{array}{l}\text { El docente de un aula inclusiva requiere co- } \\
\text { nocer estrategias pedagógicas diferentes. }\end{array}$ & TA & MA & I & MD & TD \\
\hline 2 & $\begin{array}{l}\mathrm{A} 1 \\
\mathrm{~B} 2\end{array}$ & $\begin{array}{c}\text { Creo que la educación inclusiva está } \\
\text { dirigida únicamente a los estudiantes con } \\
\text { discapacidad y aquellos pertenecientes a } \\
\text { minorías étnicas. }\end{array}$ & TA & MA & 1 & MD & TD \\
\hline 3 & $\begin{array}{l}\mathrm{A} 1 \\
\mathrm{~B} 1\end{array}$ & $\begin{array}{l}\text { El éxito de la educación inclusiva depende } \\
\text { exclusivamente del docente. }\end{array}$ & TA & MA & 1 & MD & TD \\
\hline 4 & $\begin{array}{l}\text { A1 } \\
\text { B2 }\end{array}$ & $\begin{array}{l}\text { La educación inclusiva favorece el apren- } \\
\text { dizaje, la participación y la convivencia de } \\
\text { todos los estudiantes del aula. }\end{array}$ & TA & MA & I & MD & TD \\
\hline 5 & $\begin{array}{l}\text { A1 } \\
\text { B2 }\end{array}$ & $\begin{array}{l}\text { Los únicos estudiantes beneficiados en un } \\
\text { aula inclusiva, son aquellos con discapaci- } \\
\text { dad y/o dificultades de aprendizaje. }\end{array}$ & TA & MA & 1 & MD & TD \\
\hline 6 & $\begin{array}{l}\text { A1 } \\
\text { B3 }\end{array}$ & $\begin{array}{c}\text { Es más difícil el manejo de la disciplina en } \\
\text { un aula inclusiva que en un aula regular/ } \\
\text { tradicional. }\end{array}$ & TA & MA & 1 & MD & TD \\
\hline 7 & $\begin{array}{l}\text { A1 } \\
\text { B3 }\end{array}$ & $\begin{array}{l}\text { En las aulas inclusivas se trabajan conteni- } \\
\text { dos más variados y llamativos. }\end{array}$ & TA & MA & 1 & MD & TD \\
\hline 8 & $\begin{array}{l}\text { A1 } \\
\text { B4 }\end{array}$ & Todas las escuelas deberían ser inclusivas. & TA & MA & 1 & MD & TD \\
\hline 9 & $\begin{array}{l}\text { A2 } \\
\text { B1 }\end{array}$ & $\begin{array}{c}\text { Desearía poder trabajar únicamente en un } \\
\text { aula regular/tradicional. }\end{array}$ & TA & MA & 1 & $\mathrm{MD}$ & TD \\
\hline 10 & $\begin{array}{l}\text { A2 } \\
\text { B1 }\end{array}$ & $\begin{array}{l}\text { Me preocupa que en un aula inclusiva deba } \\
\text { invertir mucho tiempo en los estudiantes } \\
\text { que tienen mayores dificultades. }\end{array}$ & TA & MA & 1 & MD & TD \\
\hline 11 & $\begin{array}{l}\text { A2 } \\
\text { B3 }\end{array}$ & $\begin{array}{c}\text { Me preocupa que en un aula inclusiva no } \\
\text { alcance a dar todos los contenidos del } \\
\text { plan de estudio. }\end{array}$ & TA & MA & 1 & MD & TD \\
\hline 12 & $\begin{array}{l}\text { A2 } \\
\text { B4 }\end{array}$ & $\begin{array}{c}\text { Me inquieta que la educación inclusiva sea } \\
\text { una política educativa obligatoria. }\end{array}$ & TA & MA & 1 & MD & TD \\
\hline 13 & $\begin{array}{l}\text { A2 } \\
\text { B4 }\end{array}$ & $\begin{array}{l}\text { Me molesta que las escuelas no puedan } \\
\text { determinar de forma autónoma qué estu- } \\
\text { diantes ingresan a esta. }\end{array}$ & $\mathrm{TA}$ & MA & 1 & MD & TD \\
\hline 14 & $\begin{array}{l}\text { A2 } \\
\text { B3 }\end{array}$ & $\begin{array}{l}\text { Me preocupa que todas las aulas se trans- } \\
\text { formen en inclusivas. }\end{array}$ & $\mathrm{TA}$ & MA & 1 & MD & TD \\
\hline 15 & $\begin{array}{l}\text { A3 } \\
\text { B1 }\end{array}$ & $\begin{array}{l}\text { Estoy dispuesto a cualificarme para asumir } \\
\text { un aula inclusiva. }\end{array}$ & $\mathrm{TA}$ & MA & 1 & MD & TD \\
\hline 16 & $\begin{array}{l}\text { A2 } \\
\text { B2 }\end{array}$ & $\begin{array}{c}\text { Si los estudiantes pudieran tener acceso a } \\
\text { educación especializada de acuerdo a sus } \\
\text { características, tendrían mejores procesos } \\
\text { de aprendizaje y participación. }\end{array}$ & $\mathrm{TA}$ & MA & 1 & MD & TD \\
\hline 17 & $\begin{array}{l}\text { A3 } \\
\text { B2 }\end{array}$ & $\begin{array}{l}\text { Yo no sugeriría una escuela inclusiva para } \\
\text { un estudiante normal. }\end{array}$ & $\mathrm{TA}$ & MA & 1 & MD & TD \\
\hline 18 & $\begin{array}{l}\text { A3 } \\
\text { B3 }\end{array}$ & $\begin{array}{l}\text { Estoy dispuesto a emplear organizacio- } \\
\text { nes flexibles de aula para favorecer el } \\
\text { aprendizaje y participación de todos mis } \\
\text { estudiantes. }\end{array}$ & $\mathrm{TA}$ & MA & 1 & MD & TD \\
\hline 19 & $\begin{array}{l}\text { A3 } \\
\text { B3 }\end{array}$ & $\begin{array}{l}\text { Acondicionaría mi aula de clase para facili- } \\
\text { tar el aprendizaje y participación de todos } \\
\text { los estudiantes. }\end{array}$ & TA & MA & 1 & MD & TD \\
\hline
\end{tabular}


La educación inclusiva en docentes en formación: su evaluación a partir de la teoría de facetas

Libia Vélez Latorre

\begin{tabular}{|c|c|c|c|c|c|c|c|}
\hline & & & $\begin{array}{l}\text { Total } \\
\text { acuerdo }\end{array}$ & $\begin{array}{l}\text { Moderado } \\
\text { acuerdo }\end{array}$ & Indiferente & $\begin{array}{l}\text { Moderado } \\
\text { desacuerdo }\end{array}$ & $\begin{array}{c}\text { Total } \\
\text { desacuerdo }\end{array}$ \\
\hline 20 & $\begin{array}{l}\text { A3 } \\
\text { B4 }\end{array}$ & $\begin{array}{l}\text { Las escuelas deben implementar estrate- } \\
\text { gias de educación continua para asumir la } \\
\text { educación inclusiva. }\end{array}$ & TA & MA & 1 & MD & TD \\
\hline 21 & $\begin{array}{l}\text { A3 } \\
\text { B4 }\end{array}$ & $\begin{array}{l}\text { Las escuelas deberían tener aulas especia- } \\
\text { lizadas de acuerdo con las particularidades } \\
\text { de los estudiantes. }\end{array}$ & TA & MA & 1 & MD & TD \\
\hline 22 & $\begin{array}{l}\text { A3 } \\
\text { B4 }\end{array}$ & $\begin{array}{c}\text { Debería haber escuelas inclusivas y escue- } \\
\text { las regulares/tradicionales. }\end{array}$ & $\mathrm{TA}$ & MA & 1 & MD & TD \\
\hline 23 & $\begin{array}{l}\text { A1 } \\
\text { C1 }\end{array}$ & $\begin{array}{c}\text { En las aulas inclusivas se deben emplear } \\
\text { las mismas estrategias didácticas que en } \\
\text { un aula regular/tradicional. }\end{array}$ & TA & MA & 1 & MD & TD \\
\hline 24 & $\begin{array}{l}\text { A2 } \\
\text { C3 }\end{array}$ & $\begin{array}{c}\text { Siento que los ambientes de aprendizaje } \\
\text { no son esenciales para el desarrollo exito- } \\
\text { so de las aulas inclusivas. }\end{array}$ & TA & MA & 1 & MD & TD \\
\hline 25 & $\begin{array}{l}\text { A1 } \\
\text { C3 }\end{array}$ & $\begin{array}{l}\text { Los agrupamientos de los estudiantes de } \\
\text { formas variadas favorecen la atención a la } \\
\text { diversidad en el aula inclusiva. }\end{array}$ & TA & MA & 1 & MD & TD \\
\hline 26 & $\begin{array}{l}\mathrm{A} 2 \\
\mathrm{C} 1\end{array}$ & $\begin{array}{l}\text { Me preocupa que las estrategias nece- } \\
\text { sarias para un aula inclusiva sean muy } \\
\text { complejas y dispendiosas para desarrollar. }\end{array}$ & TA & MA & 1 & MD & TD \\
\hline 27 & $\begin{array}{l}\mathrm{A} 1 \\
\mathrm{C} 1\end{array}$ & $\begin{array}{l}\text { Estoy convencido que el empleo de estra- } \\
\text { tegias inclusivas mejora el clima del aula. }\end{array}$ & TA & MA & 1 & MD & TD \\
\hline 28 & $\begin{array}{l}\text { A1 } \\
\text { C2 }\end{array}$ & $\begin{array}{l}\text { Las escuelas no cuentan con el suficiente } \\
\text { material para atender las particularidades } \\
\text { de un aula inclusiva. }\end{array}$ & TA & MA & 1 & MD & TD \\
\hline 29 & $\begin{array}{l}\text { A2 } \\
\mathrm{C} 2\end{array}$ & $\begin{array}{c}\text { Me sentiría frustrado si la escuela no me } \\
\text { suministrara los recursos requeridos para } \\
\text { atender un aula inclusiva. }\end{array}$ & TA & MA & 1 & MD & TD \\
\hline 30 & $\begin{array}{l}\mathrm{A} 2 \\
\mathrm{C} 1\end{array}$ & $\begin{array}{l}\text { Me molesta tener que utilizar diferentes } \\
\text { formas de evaluar los procesos de aprendi- } \\
\text { zaje de mis estudiantes. }\end{array}$ & $\mathrm{TA}$ & MA & 1 & MD & TD \\
\hline 31 & $\begin{array}{l}\text { A3 } \\
\text { C1 }\end{array}$ & $\begin{array}{c}\text { Como docente de aula inclusiva seguiría } \\
\text { empleando las mismas estrategias de } \\
\text { enseñanza que conozco. }\end{array}$ & TA & MA & 1 & MD & TD \\
\hline 32 & $\begin{array}{l}\text { A3 } \\
\text { C2 }\end{array}$ & $\begin{array}{l}\text { Sin material especializado no puedo de- } \\
\text { sarrollar adecuadamente los procesos de } \\
\text { enseñanza en un aula inclusiva. }\end{array}$ & TA & MA & 1 & MD & TD \\
\hline 33 & $\begin{array}{l}\text { A3 } \\
\text { C3 }\end{array}$ & $\begin{array}{c}\text { Estaría dispuesto a trabajar por proyectos } \\
\text { de aula, rincones de aprendizaje u otra } \\
\text { organización flexible que favorezca el } \\
\text { aprendizaje y participación de todos los } \\
\text { estudiantes. }\end{array}$ & TA & MA & I & MD & TD \\
\hline
\end{tabular}

Gracias por su colaboración. 